\title{
INFLUENCE OF GEOMETRIC AND PHYSICAL NONLINEARITIES ON THE INTERNAL RESONANCES OF A FINITE CONTINUOUS ROD WITH A MICROSTRUCTURE
}

\author{
Igor V. Andrianov ${ }^{1, *}$, Jan Awrejcewicz ${ }^{2}$, Vladyslav V. Danishevskyy ${ }^{3}$, Bernd Markert $^{1}$ \\ ${ }^{1}$ Institute of General Mechanics, RWTH Aachen University, Germany \\ ${ }^{2}$ Department of Automation, Biomechanics and Mechatronics, \\ Technical University of Lodz, Poland \\ ${ }^{3}$ School of Computing and Mathematics, Keele University, UK
}

\begin{abstract}
In this work, nonlinear longitudinal vibrations of a finite composite rod are studied including geometric and physical nonlinearities. An original boundary value problem for a heterogeneous rod yielded by the macroscopic approximation obtained earlier by the higher-order asymptotic homogenization method is used. The effects of internal resonances and modes coupling are predicted, validated and analyzed. The defined novel continuous problem governed by PDEs is solved using space-discretization and the method of multiple time scales. We are aimed at understanding and analyzing how the presence of the microstructure influences the processes of mode interaction. It is shown that, depending on a scaling relation between the amplitude of the vibrations and the size of the unit cell, different scenarios of the modes coupling can be realized. Additionally to the asymptotic solution, numerical simulation of the modes coupling is performed by means of the Runge-Kutta fourth-order method. The obtained numerical and analytical results demonstrate good qualitative agreement.
\end{abstract}

Keywords: nonlinear vibrations, rod, microstructure, physical and geometric nonlinearities, dispersion, asymptotic homogenization method, space-discretization, method of multiple time scales, numerical simulation, internal resonance, energy transfers between modes

\section{Introduction}

It is well known and widely observed in engineering that nonlinear dynamical effects belong to the main factors responsible for the composite constructions strength and their long-time operating ability. It should be emphasized that nonlinear elastic moduli of rigid bodies are highly

\footnotetext{
*Corresponding author: igor_andrianov@hotmail.com (for I.V. Andrianov).
} 
sensitive to changes of microstructure patterns [1]. Experimental investigations show that nonlinear characteristics of dynamical processes are associated with internal material reconstruction on the nanoscale levels, which cannot be directly predicted by the standard methods of investigation. Full understanding and analysis of the mentioned nanoscale processes require application of novel acoustic-based diagnosis and health monitoring of the mentioned events in order to achieve a possibility of their control. On the other hand, the particular features of nonhomogeneous material objects, including wave dispersion and accumulation thresholds, selected reflection, and other, can be understood and then employed while designing novel materials dedicated to various technical applications including noise- and vibro-isolated covers, vibration dampers, acoustic filters, ultrasound sensing and amplifying, wave transmitting, etc.

For a theoretical description of the nonlinear dynamical effects in composite materials, a number of higher-order continuum models mainly focused on reduction of heterogeneous objects to the homogeneous ones were proposed, For detailed reviews of the subject, we refer to Ting [2], Engelbrecht and Braun [3], Erofeev [4], Cattani and Rushchitsky [5], and Rushchitsky [6]. The mentioned homogeneous models are widely employed in infinite spaces, which gives a possibility of application of wide palette of methods offered by modern Nonlinear Physics (inverse scattering transforms, theory of solitons, library of integrated nonlinear equations, etc., see Bhatnagar [7], Porubov [8], Samsonov [9] for more details). Surprisingly, a little is known while dealing with the spaces of finite dimensions, which are also associated with novel dynamical phenomena. The latter include chatter type reflections, which are associated with occurrence of complex boundary value problems.

The effect of internal resonances may arise in nonlinear multi-degree-of-freedom systems, when natural frequencies of the modes become commensurable with each other. Then, the presence of nonlinearity induces coupling between different modes even in zero-order approximation. Then, complicated modal interactions occur, which may result in a self-generation of higher-order modes. In such a case, truncation to the modes having nonzero initial energy (which is usually encountered while studying vibrations of continuous structures) will not be valid and all resonant modes should be taken into account simultaneously.

The nonlinear phenomena of modes coupling and internal resonances have been intensively investigated for homogeneous structures. For this purpose, either numerical [10] or asymptotic approaches [11-14] have been applied. The latter allow getting more adequate imagination of the qualitative/quantitative system dynamical behavior and this gives also a motivation for our paper. Simultaneously, the nonlinear dynamic behavior of heterogeneous solids was considered to a significantly less extent. Several studies on nonlinear vibrations of composite structures were presented in references [15-17]. However, many authors have focused on laminated plates and 
shells that include a small number of layers (usually only a few), so the influence of a microstructure was not thoroughly investigated. Only very recently, vibrations of a heterogeneous rod embedded in a nonlinear elastic medium were considered in [18].

In this paper, natural longitudinal vibrations of an elastic periodically heterogeneous rod are studied. Geometric and physical nonlinearity of the problem is taken into account. We are aimed at answering the following important question: How does the presence of the microstructure affect the processes of internal resonances and mode interactions? It should be mentioned that the governing macroscopic dynamical equation has been obtained earlier with the help of the asymptotic homogenization method [19, 20], where the method of multiple time scales has been employed for the analysis of nonlinear dynamical behavior of the rod.

The paper is organized as follows. In Section 2, the input problem is formulated. In Section 3, the perturbation procedure for a homogeneous rod is introduced. In Section 4, the influence of the microstructure is analyzed. In Section 5, we present a numerical simulation of the energy transfers between different modes. Conclusion remarks are presented in Section 6.

\section{Input problem}

We consider a periodically heterogeneous composite rod consisting of alternating layers of two different components $\Omega^{(1)}$ and $\Omega^{(2)}$ with a perfect bonding at the interface $\partial \Omega$, as it is shown in Fig. 1. Natural longitudinal vibrations in the direction $x$ are studied.

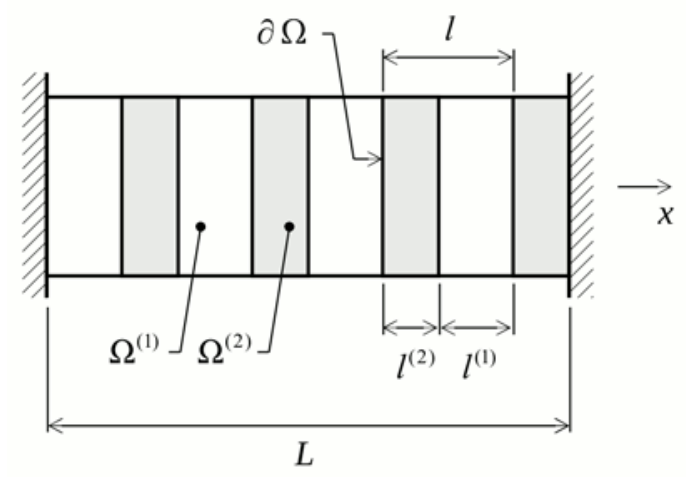

Fig. 1. Heterogeneous rod under consideration.

Structures of such type can be employed for vibration/shock control, in particular with respect to banded or band-gap structures [21]. In nanomechanics, great attention has been recently paid to 1D bilayer assemblies of nanowires or nanotubes [22]. Furthermore, the model shown in Fig. 1 can be also employed while studying the non-linear dynamics of trains, multistage rockets as well as the gas- and oil- pipelines. In the case of the lattermost, steel pipes are joined by rubber 
compensators. In many cases, the applied fitting can be modelled with the use of homogeneous rods with periodically varying cross sections. In this case, it is useful to consider first an equivalent 1D model that assumes constant cross section but varying material properties.

Geometric nonlinearity appears due to nonlinear relations between the elastic strains and the gradients of displacements and is described by the Cauchy-Green strain tensor [23]. Physical nonlinearity displays a deviation of the stress-strain relations from classical Hooke's law. It is modeled representing the energy of deformation as a series expansion in powers of invariants of the strain tensor and taking into account the higher-order terms. Nowadays, the latter series is known as the elastic potential (see Murnaghan [24]). It should be noted that the origin of this approach can be found in the work of Landau and Rumer [25], whereas the five time-points model of elasticity was proposed by Voigt in 1893 [26].

The energy of deformation $W$ of a nonlinear body can be expressed by the Murnaghan [24] elastic potential:

$$
W=\frac{1}{2} \lambda \varepsilon_{k_{1} k_{1}}^{2}+\mu \varepsilon_{k_{1} k_{2}}^{2}+\frac{1}{3} A \varepsilon_{k_{1} k_{2}} \varepsilon_{k_{1} k_{3}} \varepsilon_{k_{2} k_{3}}+B \varepsilon_{k_{1} k_{1}} \varepsilon_{k_{1} k_{2}}^{2}+\frac{1}{3} C \varepsilon_{k_{1} k_{1}}^{3}
$$

where $\varepsilon_{k_{1} k_{2}}$ are the strains,

$$
\varepsilon_{k_{1} k_{2}}=\frac{1}{2}\left(\frac{\partial u_{k_{1}}}{\partial x_{k_{2}}}+\frac{\partial u_{k_{2}}}{\partial x_{k_{1}}}+\frac{\partial u_{k_{3}}}{\partial x_{k_{1}}} \frac{\partial u_{k_{3}}}{\partial x_{k_{2}}}\right), \quad \varepsilon_{k_{1} k_{2}}=\varepsilon_{k_{2} k_{1}}, \quad k_{1}, k_{2}, k_{3}=1,2,3 .
$$

Furthermore, $u_{i}$ stand for the displacements, $\lambda, \mu$ are the Lamé elastic constants, and $A, B$, $C$ are the Landau elastic constants.

The stresses $\sigma_{k_{1} k_{2}}$ are defined as follows:

$$
\sigma_{k_{1} k_{2}}=\frac{\partial W}{\partial\left(\partial u_{k_{1}} / \partial x_{k_{2}}\right)}
$$

Observe that the expressions (1) and (2) describe the effects of physical and geometric nonlinearity, respectively.

The nonlinear dynamical equation for each of the layers $\Omega^{(i)}$ reads 


$$
\alpha_{i} \frac{\partial^{2} u^{(i)}}{\partial x_{1}^{2}}+\beta_{i} \frac{\partial u^{(i)}}{\partial x_{1}} \frac{\partial^{2} u^{(i)}}{\partial x_{1}^{2}}=\rho_{i} \frac{\partial^{2} u^{(i)}}{\partial t^{2}}
$$

where $\rho_{i}$ is the mass density, $\alpha_{i}=\lambda_{i}+2 \mu_{i}, \beta_{i}=3\left(\lambda_{i}+2 \mu_{i}\right)+2\left(A_{i}+3 B_{i}+C_{i}\right)$, the indexes $i=1,2$ denote different components of the structure.

At the interface $\partial \Omega$, we assume perfect bonding conditions that correspond to the equalities of displacements and tractions, which means that:

$$
\left.\left\{u^{(1)}=u^{(2)}\right\}\right|_{\partial \Omega},\left.\left\{\alpha_{1} \frac{\partial u^{(1)}}{\partial x_{1}}+\frac{\beta_{1}}{2}\left(\frac{\partial u^{(1)}}{\partial x_{1}}\right)^{2}=\alpha_{2} \frac{\partial u^{(2)}}{\partial x_{1}}+\frac{\beta_{2}}{2}\left(\frac{\partial u^{(2)}}{\partial x_{1}}\right)^{2}\right\}\right|_{\partial \Omega} .
$$

In $[19,20]$, for this problem, the higher-order asymptotic homogenization method was applied. The basic idea of this method can be described as follows. Let us distinguish two spatial scales: microscale, associated with the size $l$ of a periodically repeated unit cell, and macroscale, associated with the length $L$ of a travelling wave. Supposing $l<L$, we define a small parameter $\delta=l / L$ that characterizes the rate of heterogeneity of the problem. Instead of the original coordinate variable $x$, let us introduce so called slow $x_{1}=x$ and fast $\xi=\delta^{-1} x_{1}$ coordinates (for the slow coordinate, we keep the previous notation $x$ ). The derivative reads $\partial / \partial x_{1}=\partial / \partial x+\varepsilon^{-1} \partial / \partial \xi$. The displacement field $u$ is searched as the asymptotic expansion

$$
u^{(i)}\left(x_{1}, t\right)=u_{0}(x, t)+\delta u_{1}^{(i)}(x, \xi, t)+\delta^{2} u_{2}^{(i)}(x, \xi, t)+\ldots
$$

Here, the first term $u_{0}$ represents the homogeneous part of the solution; it varies slowly on the macroscale and does not depend on fast variables. The next terms $u_{j}^{(i)}, j=1,2,3, \ldots$ provide corrections of the orders $\delta^{j}$ and describe local variations of the displacements on the microscale. The spatial periodicity of the composite induces the same periodicity for $u_{j}^{(i)}$ with respect to $\xi$ : $u_{j}^{(i)}(x, \xi, t)=u_{j}^{(i)}(x, \xi+L, t)$.

Splitting the input problem (4), (5) with respect to $\delta$, we come to a recurrent sequence of cell problems considered within a unit cell of the composite structure. Solutions to the cell problems are provided by the terms $u_{j}^{(i)}$. We find them asymptotically using series expansions in powers of 
the displacement gradients $\partial u_{j}^{(i)} / \partial \xi$. Finally, performing the homogenization by the fast coordinate $\xi$ over the unit cell domain, we obtain a homogenized (so called macroscopic) dynamical equation:

$$
E_{1} \frac{\partial^{2} u}{\partial x^{2}}+E_{2} \frac{\partial u}{\partial x} \frac{\partial^{2} u}{\partial x^{2}}+l^{2} E_{3} \frac{\partial^{4} u}{\partial x^{4}}=\rho \frac{\partial^{2} u}{\partial t^{2}},
$$

where $u$ is the longitudinal displacement; $l$ is the size of the unit cell; $E_{1}, E_{2}, E_{3}$ are the effective elastic coefficients; $\rho$ is the effective mass density. In the l.h.s. of equation (6), the first term is associated with a linear elastic response of a homogeneous solid. The second term accounts for nonlinear effects (both physical and geometric). The third term describes the influence of the microstructure.

For the effective coefficients, explicit analytical expressions are derived in [20]:

$$
\begin{aligned}
& E_{1}=\frac{\alpha^{(1)} \alpha^{(2)}}{c^{(1)} \alpha^{(2)}+c^{(2)} \alpha^{(1)}}, E_{2}=\frac{c^{(1)} \beta^{(1)}\left(\alpha^{(2)}\right)^{3}+c^{(2)} \beta^{(2)}\left(\alpha^{(1)}\right)^{3}}{\left(c^{(1)} \alpha^{(2)}+c^{(2)} \alpha^{(1)}\right)^{3}}, \\
& E_{3}=\frac{1}{12}\left(c^{(1)}\right)^{2}\left(c^{(2)}\right)^{2} \frac{v_{0}^{4}}{\left(v^{(1)}\right)^{2}\left(v^{(2)}\right)^{2}}\left(\frac{z^{(1)}}{z^{(2)}}-\frac{z^{(2)}}{z^{(1)}}\right)^{2}, \rho=c^{(1)} \rho^{(1)}+c^{(2)} \rho^{(2)},
\end{aligned}
$$

where $c^{(i)}=l^{(i)} / l$ are the volume fractions of the components; $v^{(i)}=\sqrt{\alpha^{(i)} / \rho^{(i)}}$ are the velocities of propagation through each of the components in the linear case; $z^{(i)}=\sqrt{\alpha^{(i)} \rho^{(i)}}$ are the acoustic impedances of the components; the superscripts $(i), i=1,2$, refer to different components of the composite material; $\alpha^{(i)}=\lambda^{(i)}+2 \mu^{(i)} ; \beta^{(i)}=3\left(\lambda^{(i)}+2 \mu^{(i)}\right)+2\left(A^{(i)}+3 B^{(i)}+C^{(i)}\right) ; \rho^{(i)}$ is the mass density; $\lambda, \mu$ are the Lamé constants (elastic moduli of the second order), and $A, B, C$ are the Landau constants (elastic moduli of the third order).

It should be noted that analytical results for the linear effective coefficients $E_{1}, E_{3}$ are available in the existing literature [27], while the proposed solution regarding introduction of the nonlinear elastic modulus $E_{2}$ belongs to a novel input of our research.

Though the coefficients $E_{1}, E_{3}$ are always positive, $E_{2}$ is negative for the most industrial materials (it will be positive in the case of a physically linear solid). The typically used magnitudes of the elastic coefficients are as follows: $\left|E_{2}\right| / E_{1} \sim 10, E_{3} / E_{1} \sim 10^{-2}$. 
Equation (6) presents an asymptotic approximation of the original problem. It is valid only if the size of the microstructure $l$ is smaller than the macroscopic size $L$ of the entire body, $l / L<1$. In reference [20], it was shown that good accuracy of the asymptotic approach is achieved for $l / L<0.4$, i.e., $l^{2} / L^{2} \leq 10^{-1}$.

Let us introduce non-dimensional variables $\bar{x}=x(\pi / L), \bar{t}=t(\pi / L) \sqrt{E_{1} / \rho}, \bar{u}=u / A$, where $A$ is the amplitude of the vibrations. For the simplicity, in the sequel, the over bars are omitted. Then, equation (6) takes the following nondimensional form

$$
\frac{\partial^{2} u}{\partial x^{2}}+\varepsilon \frac{\partial u}{\partial x} \frac{\partial^{2} u}{\partial x^{2}}+\eta \frac{\partial^{4} u}{\partial x^{4}}=\frac{\partial^{2} u}{\partial t^{2}}
$$

where $\varepsilon=\pi\left(E_{2} / E_{1}\right)(A / L), \eta=\pi^{2}\left(E_{3} / E_{1}\right)\left(l^{2} / L^{2}\right)$.

Let us note that $\eta$ is always positive, whereas the sign of $\varepsilon$ depends on the properties of the material: $\varepsilon<0$ if $E_{3}<0$ (soft nonlinearity) and $\varepsilon>0$ if $E_{3}>0$ (hard nonlinearity). The variables $\eta$ and $\varepsilon$ may be considered as natural small parameters characterizing, accordingly, the rate of heterogeneity and the rate of nonlinearity.

Let us consider the case of clamped-clamped edges. The boundary and the initial conditions follow

$$
\begin{aligned}
& u(0, t)=u(\pi, t)=0, \\
& u(x, \mathrm{t})=U_{0}(x), \partial u(x, \mathrm{t}) / \partial t=U_{1}(x) \text { at } \mathrm{t}=0 .
\end{aligned}
$$

It should be noted that equation (7) includes the fourth-order spatial derivative and, consequently, additional boundary conditions are needed. This is a typical difficulty that arises when higher-order models, derived originally for infinite media, are applied to bounded domains. In reference [28], this problem has been studied for a linear mass chain, when exact solution can be easily constructed. In particular, it has been shown that for the optimization needs regarding the most suitable frequencies and modes approximation, the following additional boundary conditions should be employed

$$
\partial^{2} u(x, t) / \partial x^{2}=0 \text { at } x=0, \pi .
$$


Motivated by the abovementioned description, we have also chosen the boundary conditions (10) in our investigations.

\section{Vibrations of a homogeneous rod}

Behavior of the nonlinear problem (7)-(10) depends on the scaling relation between the small parameters $\eta$ and $\varepsilon$, which, in turn, is determined by the size $l$ of the microstructure and by the amplitude $A$ of the vibrations. In order to estimate a relation between the small parameters $\eta$ and $\varepsilon$, the following parameter $p$ is introduced: $\eta \square|\varepsilon|^{p}$. It is clear that one may deal with the following three cases, i.e., $p<1, p>1$ and $p=1$. In the first case, the system can be linearized; in the second, the influence of dispersion can be neglected. The last case is of more interest, since both nonlinearity and dispersion can be included in the investigation.

The small parameters $\delta, \eta, \varepsilon$ have the following scaling: $\delta \square l / L, \eta \square l^{2} / L^{2}$ and, at $p=1, \varepsilon \square \eta$. Therefore, $\delta^{2} \square \varepsilon$. We keep expansion (5a) up to the order $\delta^{3}$ and neglect the terms of the order $\delta^{4} \square \varepsilon^{2}$. In equation (7), we keep the term of the order $\varepsilon$ and neglect $\varepsilon^{2}$. Thus, it can be seen that the developed asymptotic procedure is consistent and non-contradictory. This also corresponds to the results shown by Fish and Chen [27].

In what follows, we consider the case when the size of the microstructure is considerably small $(p>1)$. Up to $O\left(|\varepsilon|^{p}\right)$ approximation, the influence of the microstructure can be neglected, and hence equation (7) takes the following form

$$
\frac{\partial^{2} u}{\partial x^{2}}+\varepsilon \frac{\partial u}{\partial x} \frac{\partial^{2} u}{\partial x^{2}}=\frac{\partial^{2} u}{\partial t^{2}}
$$

In order to solve the boundary value problem (11), (8), we need to apply a discretization procedure. It can be directly solved using, for instance, FEM [10]. Equivalently, it is possible to reduce the original problem to a dimer lattice using lattice approximation for the prediction of wave motion in laminates, which has been presented in references [29, 30]. Each of the mentioned approaches possesses its own benefits and drawbacks, which will be not discussed here. In our case, it is sufficient to apply only Fourier-sine series. Let us represent the displacement $u$ as a Fouriersine expansion, which satisfies the boundary conditions (8):

$$
u(x, t)=q_{1}(t) \sin (x)+q_{2}(t) \sin (2 x)+q_{3}(t) \sin (3 x) \ldots
$$


Substituting (12) into (11), we obtain:

$$
\begin{aligned}
& \frac{d^{2} q_{1}}{d t^{2}}+\omega_{1}^{2} q_{1}+\varepsilon\left(q_{1} q_{2}+3 q_{2} q_{3}+6 q_{3} q_{4}+\ldots\right)=0, \\
& \frac{d^{2} q_{2}}{d t^{2}}+\omega_{2}^{2} q_{2}+\varepsilon\left(\frac{1}{2} q_{1}^{2}+3 q_{1} q_{3}+8 q_{2} q_{4}+\ldots\right)=0, \\
& \frac{d^{2} q_{3}}{d t^{2}}+\omega_{3}^{2} q_{3}+\varepsilon\left(3 q_{1} q_{2}+6 q_{1} q_{4}+\ldots\right)=0, \\
& \frac{d^{2} q_{4}}{d t^{2}}+\omega_{4}^{2} q_{4}+\varepsilon\left(6 q_{1} q_{2}+4 q_{2}^{2}+\ldots\right)=0, \\
& \ldots ;
\end{aligned}
$$

where $\omega_{n}$ is the frequency in the linear case, $\omega_{n}=n$, whereas $n$ stands for the number of the mode, $n=1,2,3, \ldots$

The obtained system regarding the unknown function with odd and even number of the vibration mode is nonsymmetric. We do not violate a general aspect of our approach if we assume all functions with odd numbers equal to zero so that the remaining system will be described only by the functions with even mode numbers. It should be emphasized that it cannot be done vice versa, i.e., with respect to the functions with odd numbers due to the employed squared character of the nonlinearity.

Let us introduce different time scales $t_{0}=t, t_{1}=\varepsilon t$ and let us represent $q_{n}$ as an asymptotic expansion in powers of $\varepsilon$ :

$$
q_{n}(t)=q_{n 0}\left(t_{0}, t_{1}\right)+\varepsilon q_{n 1}\left(t_{0}, t_{1}\right)+O\left(\varepsilon^{2}\right) .
$$

We note that $d^{2} / d t^{2}=\partial^{2} / \partial t_{0}^{2}+2 \varepsilon \partial^{2} /\left(\partial t_{0} \partial t_{1}\right)+O\left(\varepsilon^{2}\right)$.

Next, we substitute expressions (14) into equations (13) and collect the coefficients at equal powers of $\varepsilon$. In $O\left(\varepsilon^{0}\right)$ approximation we obtain

$$
q_{n 0}=a_{n}\left(t_{1}\right) \cos \left(\omega_{n} t_{0}\right)+b_{n}\left(t_{1}\right) \sin \left(\omega_{n} t_{0}\right)
$$

where $a_{n}(0)=(2 / \pi) \int_{0}^{\pi} U_{0}(x) \sin (n x) d x, b_{n}(0)=\left[2 /\left(\pi \omega_{n}\right)\right] \int_{0}^{\pi} U_{1}(x) \sin (n x) d x$. 
Consequently, employing the $O\left(\varepsilon^{1}\right)$ approximation to equations (13) yields

$$
\begin{aligned}
& \frac{\partial^{2} q_{11}}{\partial t_{0}^{2}}+\omega_{1}^{2} q_{11}=-2 \frac{\partial^{2} q_{10}}{\partial t_{0} \partial t_{1}}-q_{10} q_{20}-3 q_{20} q_{30}-6 q_{30} q_{40}+\ldots, \\
& \frac{\partial^{2} q_{21}}{\partial t_{0}^{2}}+\omega_{2}^{2} q_{21}=-2 \frac{\partial^{2} q_{20}}{\partial t_{0} \partial t_{1}}-\frac{1}{2} q_{10}^{2}-3 q_{10} q_{30}-8 q_{20} q_{40}+\ldots, \\
& \frac{\partial^{2} q_{31}}{\partial t_{0}^{2}}+\omega_{3}^{2} q_{31}=-2 \frac{\partial^{2} q_{30}}{\partial t_{0} \partial t_{1}}-3 q_{10} q_{20}-6 q_{10} q_{40}+\ldots, \\
& \frac{\partial^{2} q_{41}}{\partial t_{0}^{2}}+\omega_{4}^{2} q_{41}=-2 \frac{\partial^{2} q_{40}}{\partial t_{0} \partial t_{1}}-6 q_{10} q_{30}-4 q_{20}^{2}+\ldots, \\
& \ldots .
\end{aligned}
$$

Straightforward integration of system (16) will lead to the appearance of secular terms in the expressions for $q_{n 1}$. Secular terms grow without a bound in time, which is inconsistent with the physical properties of the conservative system under consideration. In order to eliminate secular terms, the coefficients of $\cos \left(\omega_{n} t_{0}\right)$ and $\sin \left(\omega_{n} t_{0}\right)$ in the r.h.s. of equations (16) must be equal to zero. Substituting expressions (15) into equations (16) and fulfilling the aforementioned condition, we obtain a system of equations for $a_{n}$ and $b_{n}$, which gives a possibility to investigate the interactions between different modes.

We note that in the problem under consideration, an infinite number of modes can be involved into resonant interactions. In this paper, we consider only two leading modes and examine internal resonance between the modes 1 and 2 in detail the. The following reasons clarify and motivate our approach:

1. We employ the homogenized model, whose area of application is limited to the lower part of the frequency spectrum. The mentioned reasons limit our consideration to the first modes only.

2. As it will be shown in Section 5, excitation of the higher-order modes is greatly suppressed by the dispersion effects.

3. The carried out numerical experiments reported in Section 5 show that even if the dispersion factors are not taken into account, the obtained results are valid for the relatively large time interval of the order $\varepsilon^{-1}$.

4. In many real structures, higher modes are essentially affected by dissipation, caused by the viscosity of the material and/or internal friction. 
For the further analysis, it is convenient to introduce polar coordinates as follows: $a_{n}=r_{n} \cos \left(\varphi_{n}\right), b_{n}=r_{n} \sin \left(\varphi_{n}\right)$, where $r_{n}$ is the amplitude and $\varphi_{n}$ is the phase. After routine transformations, the condition of the elimination of secular terms gives:

$$
\begin{aligned}
& \frac{d r_{1}}{d t_{1}}=\frac{1}{4} r_{1} r_{2} \sin \left(\varphi_{2}-2 \varphi_{1}\right), \\
& r_{1} \frac{d \varphi_{1}}{d t_{1}}=-\frac{1}{4} r_{1} r_{2} \cos \left(\varphi_{2}-2 \varphi_{1}\right), \\
& \frac{d r_{2}}{d t_{1}}=-\frac{1}{16} r_{1}^{2} \sin \left(\varphi_{2}-2 \varphi_{1}\right), \\
& r_{2} \frac{d \varphi_{2}}{d t_{1}}=-\frac{1}{16} r_{1}^{2} \cos \left(\varphi_{2}-2 \varphi_{1}\right) .
\end{aligned}
$$

We consider particular solutions to the system (17)-(20). We begin with the case $r_{1}=0$, $r_{2} \neq 0$. The system (17)-(20) yields $r_{2}\left(t_{1}\right)=r_{2}(0), \varphi_{2}\left(t_{1}\right)=\varphi_{2}(0)$, i.e., the amplitude and the phase of the second mode are constant in time. This is true up to the order $\varepsilon$ for the timescale of order $\varepsilon^{-1}$. If we start with zero initial energy in the first mode, there will be no energy present up to the order $O(\varepsilon)$ on the timescale of order $\varepsilon^{-1}$.

It is easy to verify that $r_{1} \neq 0, r_{2}=0$ is impossible. It means that vibrations by a single mode 1 are not possible. If initial energy presents the mode 1, energy transfer occurs between the modes 1 and 2. Thereby, the modes 1 and 2 are coupled in the main (order of $\varepsilon^{0}$ ) approximation. This effect is called the internal resonance.

Let us study this case in more detail. Multiplying equation (11) by $r_{1}$ and equation (13) by $r_{2}$, adding both equations and performing the integration, one obtains $r_{1}^{2}+4 r_{2}^{2}=E$. This formula represents the energy conservation law, where $E$ is the constant of integration having a physical sense of the full energy of the vibrations, $E>0$. Since in the input nondimensional equation (2) the displacement $u$ has been normalized to the amplitude $A$ of the vibrations, without loss of generality, we let $E=1$. Then, equations (11)-(14) can be written as follows:

$$
\begin{aligned}
& \frac{d r_{2}}{d t_{1}}=\frac{4 r_{2}^{2}-1}{16} \sin (\psi), \\
& \frac{d \psi}{d t_{1}}=\frac{12 r_{2}^{2}-1}{16 r_{2}} \cos (\psi) ;
\end{aligned}
$$


where $\psi=\varphi_{2}-2 \varphi_{1}, 0 \leq r_{2} \leq 1 / 2$.

We numerically integrate system (21) by the Runge-Kutta fourth-order method. All numerical evaluations presented in the paper are performed in the open-source CAS Maxima using in-built convergent-proved subroutines. Practical convergence of the solution is additionally verified by decreasing twice the step of the integration and checking that this will not affect the obtained numerical data.

In Fig. 2, the results are presented in $\left(r_{2}, \psi\right)$ phase plane. The solution is $2 \pi$-periodic in $\psi$, and the parts of the phase diagram at $r_{2}>0$ and at $r_{2}<0$ are symmetric with respect to the line $r_{2}=0$. The critical points are located at $r_{2}= \pm \sqrt{3} / 6, \psi= \pm \pi m$ (centers) and at $r_{2}= \pm 1 / 2$, $\psi=\pi / 2 \pm \pi m$ (saddles); $m=0,1,2, \ldots$. We can observe that the system oscillates around an equilibrium state with a periodic energy transfer between the modes 1 and 2.

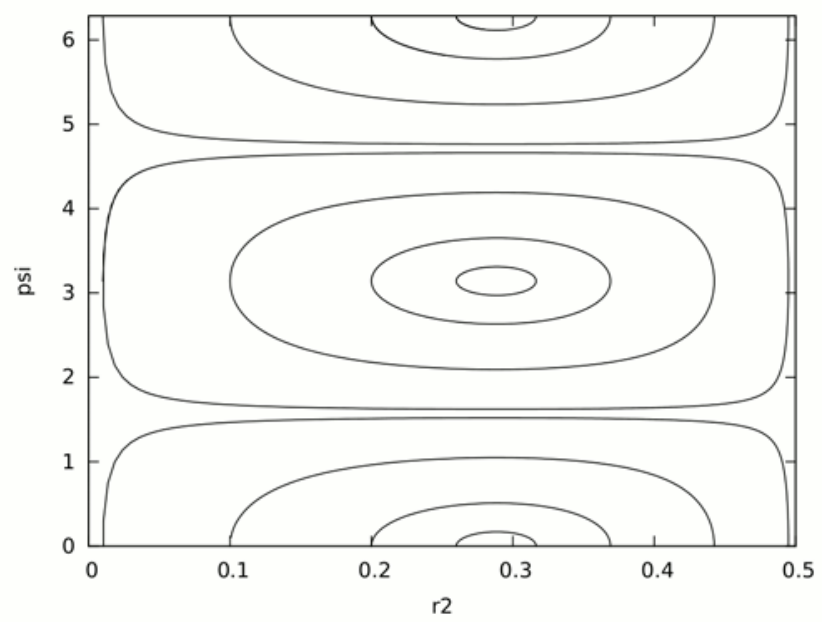

Fig. 2. Phase plane in the case of a homogeneous rod.

\section{Influence of the microstructure}

As the size of the microstructure increases, the parameters $\eta$ and $\varepsilon$ become of the same order of magnitude: $\eta \sim \varepsilon$. In such a case, $I^{2} / L^{2} \sim 10^{-1}$ for $A / L \sim 10^{-4}$. The presence of the microstructure provides a kind of detuning effect for the phenomenon of internal resonance. Let us introduce the detuning parameter $\gamma$ of the order $O(1)$ as follows: $\gamma=\eta / \varepsilon=\pi\left(E_{3} / E_{2}\right)\left(I^{2} / L^{2}\right)(L / A)$. The input dynamical equation (7) takes the form: 


$$
\frac{\partial^{2} u}{\partial x^{2}}+\varepsilon \frac{\partial u}{\partial x} \frac{\partial^{2} u}{\partial x^{2}}+\varepsilon \gamma \frac{\partial^{4} u}{\partial x^{4}}=\frac{\partial^{2} u}{\partial t^{2}}
$$

Following the asymptotic procedure presented in Section 3, we obtain an additional contribution in system (21), which now reads

$$
\begin{aligned}
& \frac{d r_{2}}{d t_{1}}=\frac{4 r_{2}^{2}-1}{16} \sin (\psi), \\
& \frac{d \psi}{d t_{1}}=\frac{12 r_{2}^{2}-1}{16 r_{2}} \cos (\psi)+3 \gamma .
\end{aligned}
$$

Let us examine the solution in the domain $0 \leq r_{2} \leq 1 / 2,0 \leq \psi \leq 2 \pi$ only because for other values of $r_{2}$ and $\psi$ it can be continued periodically. In the case of a soft nonlinearity, $\gamma<0$, examples of the phase plane are shown in Fig. 3. As $|\gamma|$ increases, one center moves to the left along the line $\psi=\pi$, whereas two centers move to the right along the lines $\psi=0, \psi=2 \pi$. One saddle moves up and the other saddle moves down along the line $r_{2}=1 / 2$. For $\gamma=-1 / 12$, centers and saddles coincide at the points $r_{2}=1 / 2, \psi=0$ and $r_{2}=1 / 2, \psi=2 \pi$ and then disappear. The only one center remains and, with the further increase in $|\gamma|$, it continues moving to the left along the line $\psi=\pi$. The area of the periodic energy transfers between the modes 1 and 2 narrows.

In the case of a hard nonlinearity, $\gamma>0$, behavior of the system is illustrated in Fig. 4. As $\gamma$ increases, one center moves to the right right along the line $\psi=\pi$ and two centers move to the left left along the lines $\psi=0, \psi=2 \pi$. For $\gamma=1 / 12$, one center and two saddles coincide at the point $r_{2}=1 / 2, \psi=\pi$ and disappear. With the further increase in $\gamma$, two centers continue moving to the left along the lines $\psi=0, \psi=2 \pi$ and the intensity of the energy transfer between the modes decreases.

For both the soft and hard nonlinearity, the increase in the size of the microstructure suppresses the energy exchange between the modes, so the effect of internal resonance becomes negligible. 


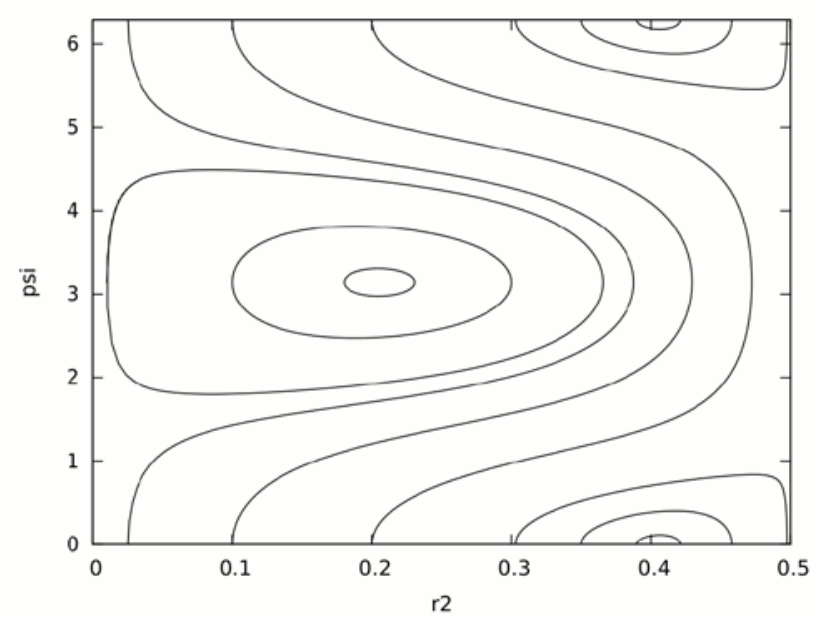

$$
\gamma=-0.05
$$

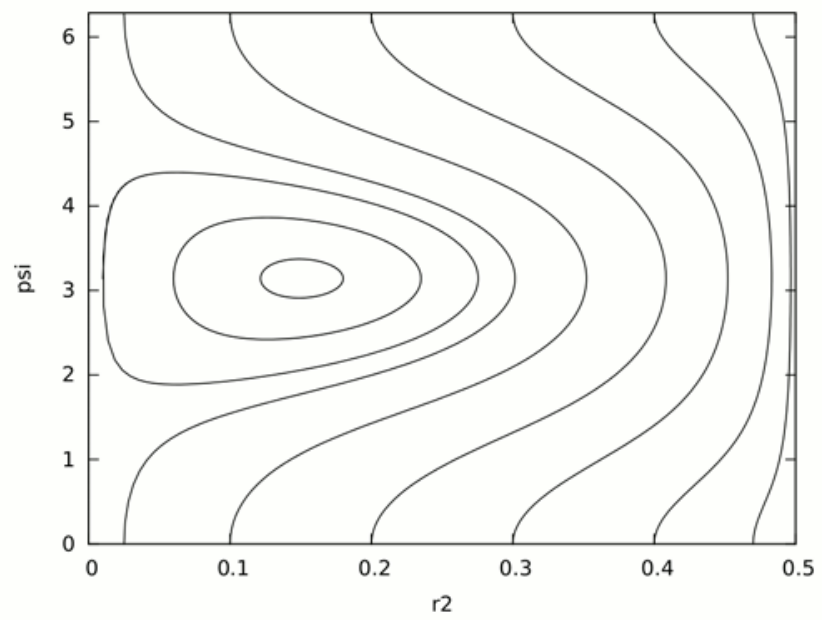

$$
\gamma=-0.1
$$

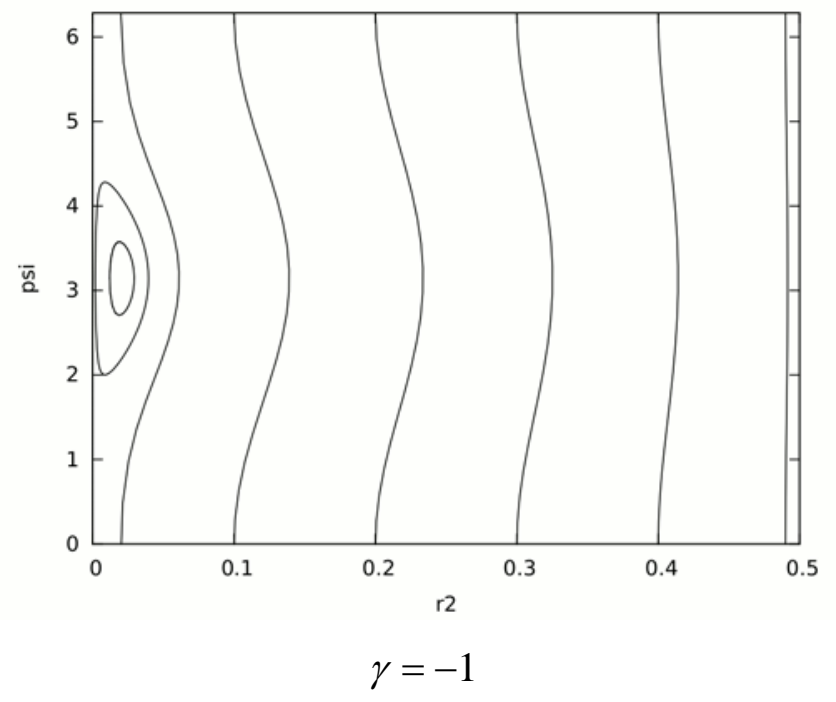

Fig. 3. Phase plane in the case of soft nonlinearity. 


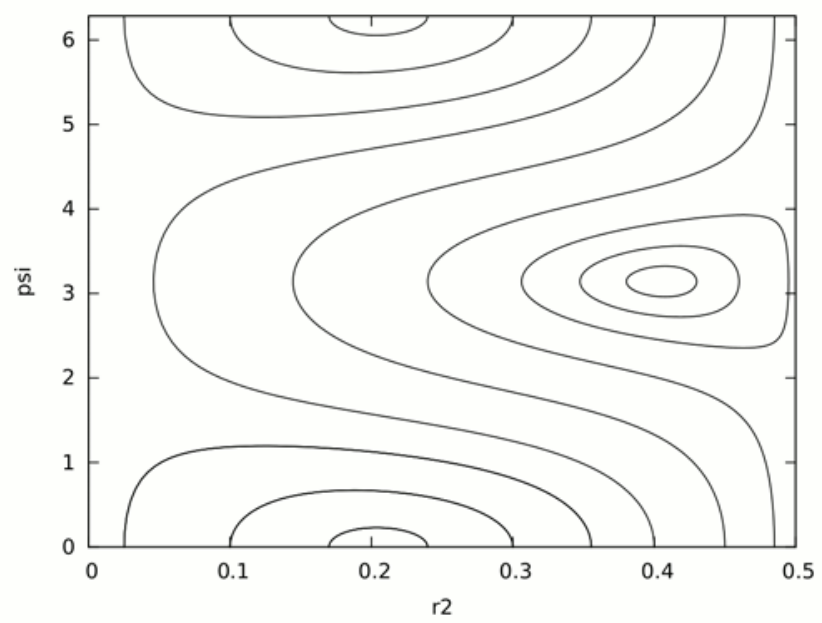

$\gamma=0.05$

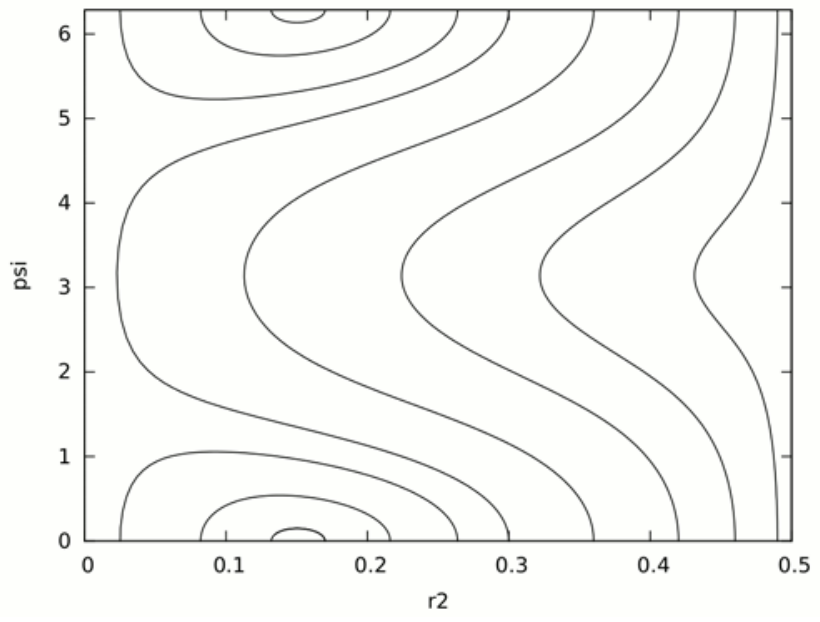

$\gamma=0.1$

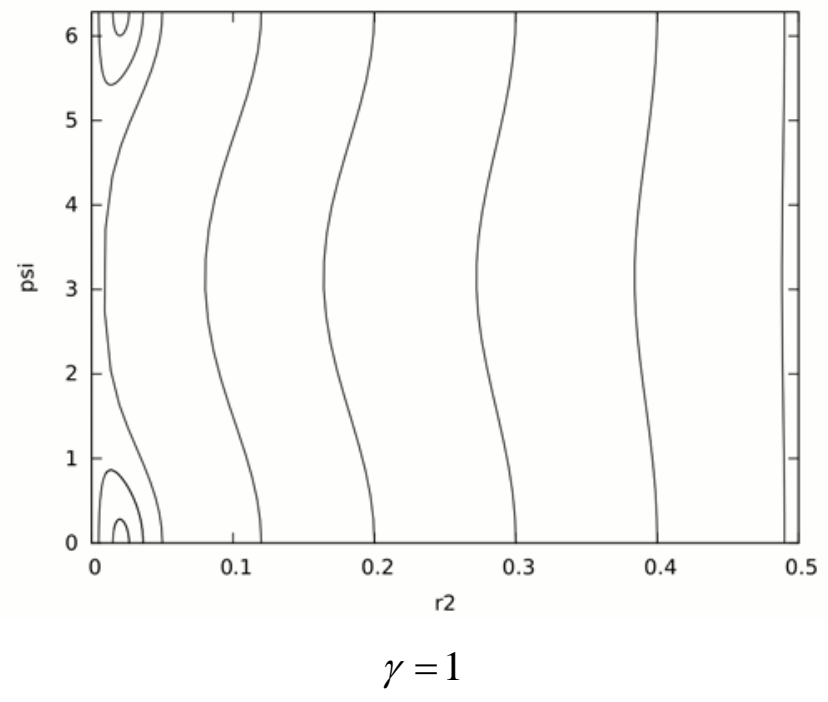

Fig. 4. Phase plane in the case of hard nonlinearity. 


\section{Numerical simulation of the modes coupling}

Now, we turn to numerical simulation of the energy transfer between different modes. The kinetic $W^{(k)}$ and the potential $W^{(p)}$ energy of the rod can be described as follows:

$$
\begin{aligned}
& W^{(k)}=\int_{0}^{\pi}\left(\frac{\partial u}{\partial t}\right)^{2} d x, \\
& W^{(p)}=\frac{1}{2} \int_{0}^{\pi}\left[\left(\frac{\partial u}{\partial x}\right)^{2}+\frac{2 \varepsilon}{3}\left(\frac{\partial u}{\partial x}\right)^{3}\right] d x .
\end{aligned}
$$

Then, taking into account expansion (6), for the full energy $W_{n}$ of the $n$-th mode, we easily obtain:

$$
W_{n}=\frac{\pi}{2}\left[\left(\frac{d q_{n}}{d t}\right)^{2}+n^{2} q_{n}^{2}\right]
$$

It is interesting to note that the second (nonlinear) term in expression (23) does not contribute to formula (24). The problem under consideration is quadratically nonlinear. Therefore, a contribution of the nonlinear term to the potential energy is of the same magnitude but opposite signs for extended and for compressed parts of the rod. The boundary conditions of the clampedclamped edges imply the Fourier-sine expansion of the displacement field. Then, after integration over the rod length, the contribution of nonlinearity is eliminated and expression (24) for the full energy is the same as it would be in the linear case. Without loss of generality, we can omit in formula (24) the coefficient $\pi / 2$ and in the further evaluations let $W_{n}=\left(d q_{n} / d t\right)^{2}+n^{2} q_{n}^{2}$.

In all numerical examples presented below, we consider a soft nonlinearity (which is typical for the most industrial materials) and set $\varepsilon=-0.1$. The increase in the magnitude of $\varepsilon$ makes the energy transfers faster; however, the qualitative behavior of the solution remains the same. Numerical integration is performed by the Runge-Kutta fourth-order method.

Firstly, let us consider vibrations of the purely homogeneous rod. The initial conditions are taken as follows:

$$
q_{1}=1, \quad q_{2}=0, \quad \frac{d q_{1}}{d t}=\frac{d q_{2}}{d t}=0 \text { at } t=0,
$$

which means that all the initial energy is localized in the first mode, whereas the initial energy of the mode 2 is zero. If we truncate system (13) to the first two equations, a periodic energy 
exchange between the modes 1 and 2 is observed (Fig. 5). Thus, the behavior of the structure is in agreement with the analytical results obtained in Section 3 (see Fig. 2).

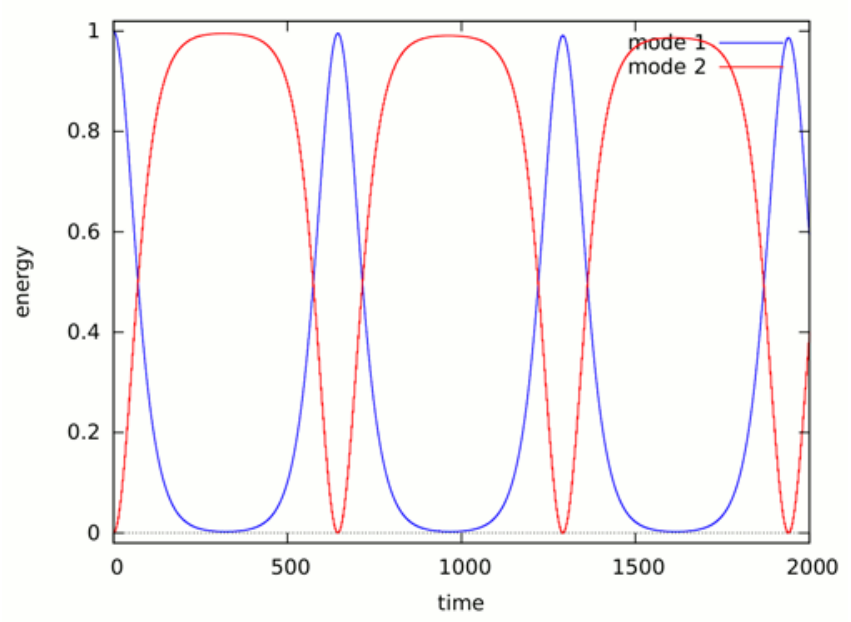

Fig. 5. Energy transfer between two leading modes in the homogeneous rod.

We should emphasize that despite the asymptotic solution developed in Section 3 is formally valid only at a relatively short timescale $t \square O\left(\varepsilon^{-1}\right)$, it is possible to provide a correct prediction for much longer times. To illustrate this, in Fig. 6 we present the results for the energy of the mode 2 . The blue curve corresponds to direct numerical integration of the original system (13) (keeping only the two first equations). The red curve corresponds to the solution of system (21), i.e., it describes $O\left(\varepsilon^{0}\right)$ approximation of the method of multiple time scale. In the latter case, the energy of the mode 2 is described as $W_{2}=4 r_{2}\left(t_{1}\right)^{2}$ with $t_{1}=\varepsilon t$. Since at $r_{2}=0$ system (21) exhibits a singularity, as an illustrative example we accept the following initial conditions:

$$
\begin{aligned}
& r_{2}=0.01, \quad r_{1}=\sqrt{1-4 r_{2}^{2}} \approx 0.99979998, \quad \frac{d r_{1}}{d t}=\frac{d r_{2}}{d t}=0 ; \\
& q_{2}=r_{2}, \quad q_{1}=r_{1}, \quad \frac{d q_{1}}{d t}=\frac{d q_{2}}{d t}=0 \text { at } t=0 .
\end{aligned}
$$




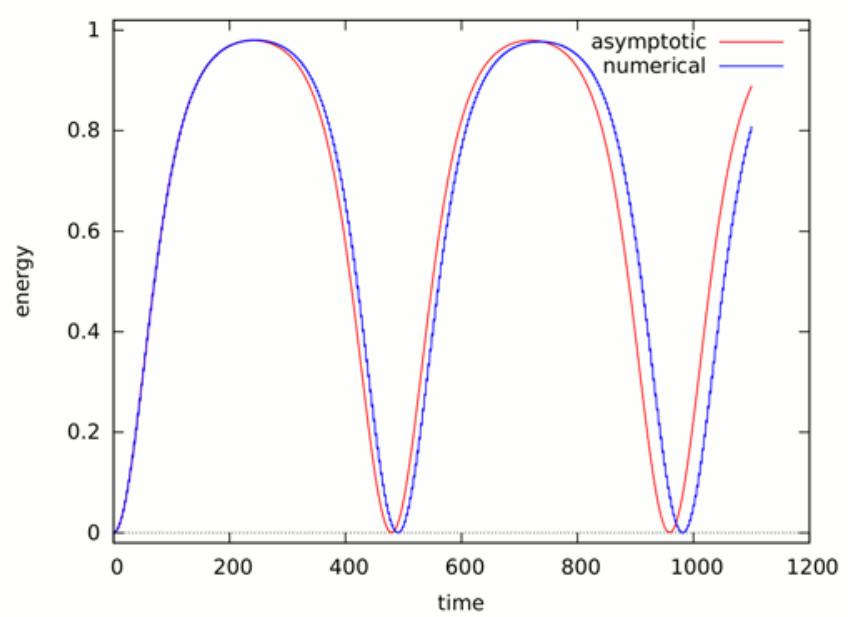

Fig. 6. Energy of the mode 2 in the two-mode approximation of the homogeneous rod.

Comparison of the asymptotic and numerical solutions.

As it was expected, accuracy of the asymptotic solution decreases in time. Nevertheless, the method of multiple time scales provides considerably good accuracy for a few first cycles of the energy exchange and, therefore, is able to give the correct qualitative prediction of the behavior of the system. We also note that if the initial energy of mode 2 increases (i.e., the magnitude of $r_{2}(0)$ in expression (26) increases), the timescale of applicability of the asymptotic solution extends.

It has been noted in Section 3 that an infinite number of modes can be involved into the resonant interactions. Fig. 7 displays energy transfers between the modes 1, 2, 3, and 4, evaluated by the truncation of system (13) to the four first equations. Again, the initial conditions correspond to the case when all the initial energy is localized in the mode 1:

$$
q_{1}=1, \quad q_{2}=q_{3}=q_{4}=0, \quad \frac{d q_{k}}{d t}=0, \quad k=\overline{1,4} \quad \text { at } t=0 .
$$

From a theoretical standpoint, higher-order modes will be generated infinitively in time and the energy will be continuously pumped to the high-frequency part of the spectrum. However, this cannot be observed in real structures, where nonlinearity is compensated by dispersion and/or dissipation. 


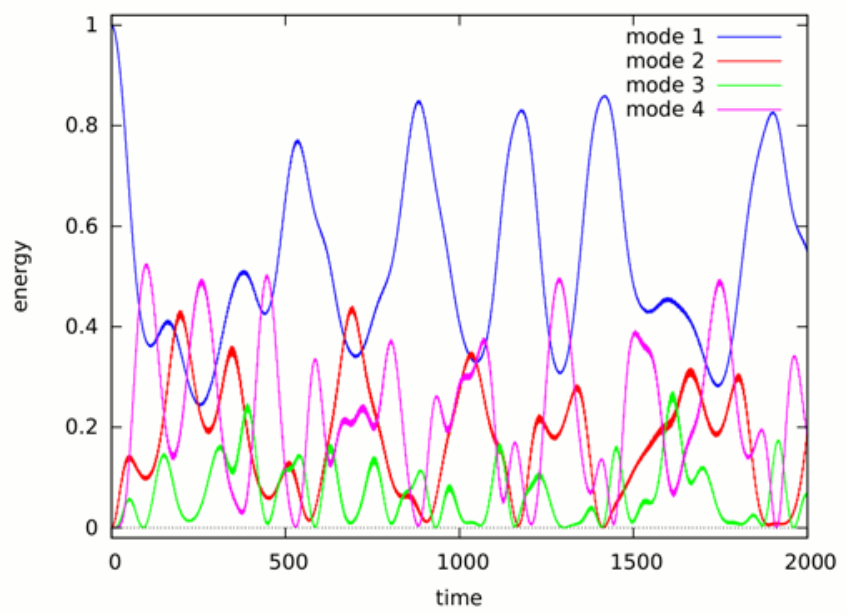

Fig. 7. Energy transfers between four leading modes in the homogeneous rod.

The presence of a microstructure leads to the dispersion effects. In order to analyze vibrations of the heterogeneous rod, we scale the small parameters in equation (7) as follows $\eta \sim \varepsilon$ and let $\gamma=\eta / \varepsilon \square O(1)$. Then, a few additional terms appear in system (13), whose first four equations now read

$$
\begin{aligned}
& \frac{d^{2} q_{1}}{d t^{2}}+\omega_{1}^{2} q_{1}+\varepsilon\left(q_{1} q_{2}+3 q_{2} q_{3}+6 q_{3} q_{4}-\gamma q_{1}\right)=0, \\
& \frac{d^{2} q_{2}}{d t^{2}}+\omega_{2}^{2} q_{2}+\varepsilon\left(\frac{1}{2} q_{1}^{2}+3 q_{1} q_{3}+8 q_{2} q_{4}-16 \gamma q_{2}\right)=0, \\
& \frac{d^{2} q_{3}}{d t^{2}}+\omega_{3}^{2} q_{3}+\varepsilon\left(3 q_{1} q_{2}+6 q_{1} q_{4}-81 \gamma q_{3}\right)=0, \\
& \frac{d^{2} q_{4}}{d t^{2}}+\omega_{4}^{2} q_{4}+\varepsilon\left(6 q_{1} q_{2}+4 q_{2}^{2}-256 \gamma q_{4}\right)=0 .
\end{aligned}
$$

The results of the numerical integration of system (28) with the initial conditions (27) are displayed in Fig. 8. Even a relatively small dispersion, $\gamma=-0.1$, leads to a significant restriction of energy transfers to the higher-order modes. As the spatial period of the mode is shorter, it is more strongly affected by the dispersion. Therefore, the higher-order modes exhibit nearly linear behavior. As it can be seen from Fig. $8 a$, in such a case, truncation to only two leading modes may provide reasonable approximation. As the size of the microstructure increases, the dispersion grows and the energy exchanges between different modes become almost negligible (see Fig. $8 b$ for $\gamma=-0.3$ ). This observation agrees with the results of the asymptotic modelling presented in Section 4. 


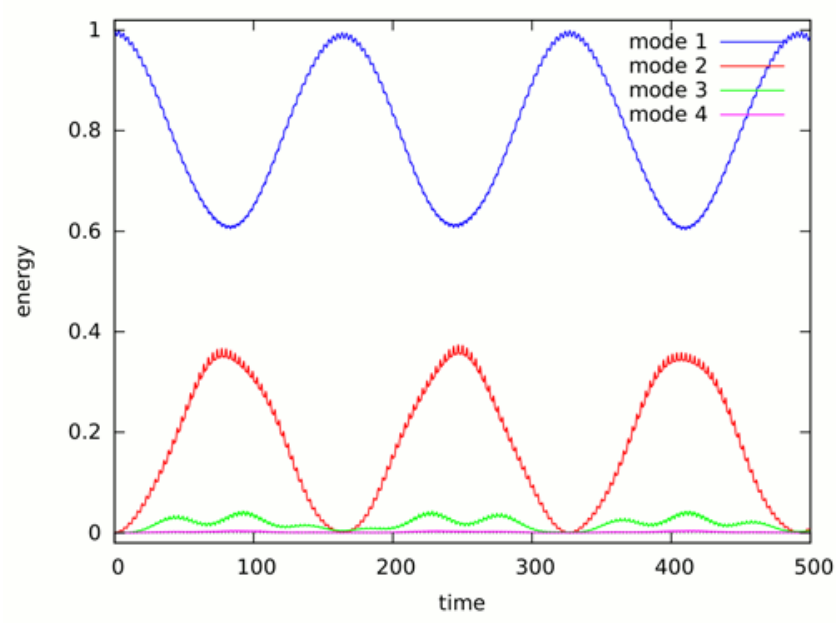

a) $\gamma=-0.1$

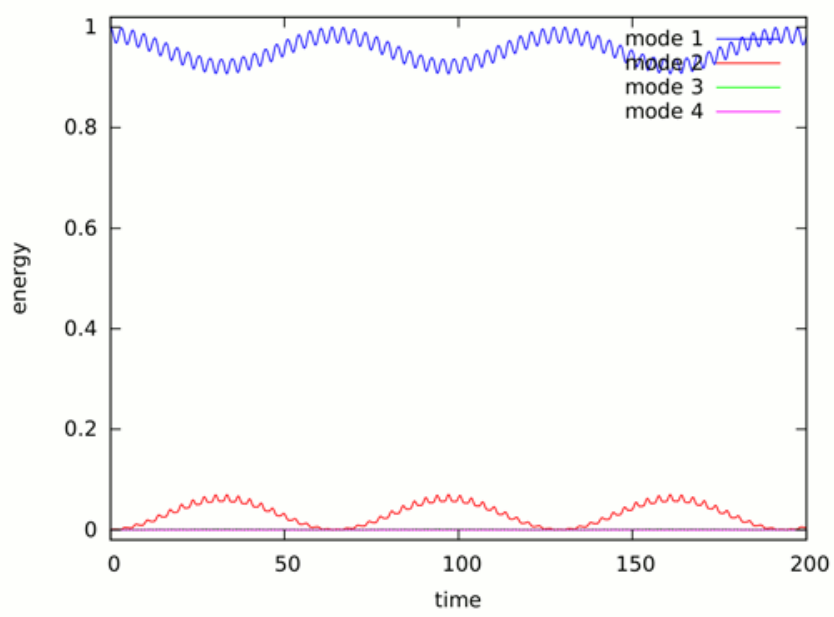

b) $\gamma=-0.3$

Fig. 8. Energy transfers in the heterogeneous rod.

\section{Conclusions}

Natural vibrations of a periodically heterogeneous rod are considered with an account for geometric and physical nonlinearity. The governing dynamical equation was obtained earlier by the method of classic higher-order asymptotic homogenization method [19, 20]. In this paper, the asymptotic analysis of the problem with the help of the method of multiple time scales is employed. If the size of the microstructure is relatively small in comparison to the amplitude of the vibrations, the essential effect of internal resonance takes place. It results in periodic energy transfers between different modes and in a modulation of their amplitudes. The resonant modes are coupled in $O\left(\varepsilon^{0}\right)$ approximation, so the truncation to the modes having nonzero initial energy is not possible. We have studied in details the internal resonance between the leading modes 1 and 2, which is of primary importance for the engineering practice. 
If the size of the microstructure increases, the dispersion supresses the influence of nonlinearity. The energy transfers to higher-order modes are restricted, so truncation to only a few leading modes can provide a reasonable approach. The further increase in the dispersion eliminates the energy exchange between the modes and the internal resonances become negligible.

Apart from the asymptotic solution, numerical simulation of the modes coupling was performed by the Runge-Kutta fourth-order. The obtained numerical and analytical results demonstrate good qualitative agreement.

It should be also mentioned that we have omitted the damping effects. On the other hand, inclusion of damping results in fast damping to the higher vibration modes, and hence the interactions are also damped (they play an important role only in the initial part of the vibrational process). Furthermore, owing to the results reported in reference [31], the modes are damped following the rule $\exp \left(-n^{2} \beta\right)$, where $n$ denotes the vibration mode number, whereas $\beta>0$ is the parameter responsible for the rod damping properties.

The results presented in the paper can be applied to facilitate the development of new efficient methods of nondestructive testing. Measuring the characteristics of nonlinear vibrations at different amplitudes allows us to receive precise information about the internal structure of heterogeneous solids. This sometimes that may be not possible within a linear framework.

Changing properties of the microstructure (e.g., using piezoelectric effects or saturation/desaturation of porous media) makes it possible to tune the macroscopic dynamic response of nonlinear structures. This can be useful for a design of new active control devices in various branches of engineering.

The effect of internal resonance may be applied for the purposes of vibration damping. Nonlinear coupling between the vibrating modes may help to transfer mechanical energy from lowto high-order modes and, therefore, to essentially decrease the amplitude of the vibrations.

Finally, it has been mentioned in the text body, that our approach does not allow taking into account the dissipation effects. This can be important, since internal resonances and energy transmission in vicinity of the first Brillouin zone of linear oscillations can be studied. This requires, however, application of the method of higher frequency homogenization [32-34] already in the beginning of the asymptotic procedure. Then, the algorithm of the asymptotic steps, described in our work, can be directly applied to solve more real world oriented problems with dissipative factors.

\section{Acknowledgments}


This work has received funding from the European Union's Horizon 2020 research and innovation program under the Marie Sklodowska-Curie grant agreement no. 655177 (for V.V. Danishevskyy).

\section{References}

1. V.Yu. Zaitsev, V.E. Nazarov, V.I. Talanov, "Nonclassical" manifestations of microstructureinduced nonlinearities: new prospects for acoustic diagnostics, Physics-Uspekhi 49 (2006) 8994.

2. T.C.T. Ting, Dynamic response of composites, Applied Mechanics Review 33 (1980) 16291635.

3. J. Engelbrecht, M. Braun, Nonlinear waves in nonlocal media, Applied Mechanics Review 51 (1998) 475-487.

4. C. Cattani, J. Rushchitsky, Wavelet and Wave Analysis as Applied to Materials with Micro or Nanostructure, World Scientific, Singapore, 2007.

5. J. Rushchitsky, Nonlinear Elastic Waves in Materials, Springer-Verlag, Berlin, 2014.

6. V.I. Erofeev, Wave Processes in Solids with Microstructure, World Scientific, Singapore, 2003.

7. P.I. Bhatnagar, Nonlinear Waves in One-Dimensional Dispersive Systems, Clarendon Press, Oxford, 1979.

8. A.V. Porubov, Amplification of Nonlinear Strain Waves in Solids, World Scientific, Singapore, 2003.

9. A.M. Samsonov, Strain Solitons in Solids and How to Construct Them, CRC Press, Boca Raton, 2001.

10. P. Ribeiro, M. Petyt, Non-linear vibration of beams with internal resonances by the hierarchical finite-element method, Journal of Sound and Vibration 224 (1999) 591-624.

11. A.I. Manevich, L.I. Manevitch, The Mechanics of Nonlinear Systems with Internal Resonances, World Scientific, Singapore, 2005.

12. A.H. Nayfeh, D.T. Mook, Nonlinear Oscillations, John Wiley and Sons, Inc, New York, 1979.

13. G.J. Boertjens, W.T. van Horssen, On mode interactions for a weakly nonlinear beam equation, Nonlinear Dynamics 17 (1998) 23-40.

14. G.J. Boertjens, W.T. van Horssen, An asymptotic theory for a weakly nonlinear beam equation with a quadratic perturbation, SIAM Journal on Applied Mathematics 60 (2000) 602-632.

15. M. Amabili, Internal resonances in non-linear vibrations of a laminated circular cylindrical shell, Nonlinear Dynamics 69 (2012) 755 -770. 
16. G. Rega, E. Saetta, Nonlinear curvature-based model and resonant finite-amplitude vibrations of symmetric cross-ply laminates, Journal of Sound and Vibration 331 (2012) 2836-2855.

17. H. Arvin, F. Bakhtiari-Nejad, Nonlinear modal interaction in rotating composite Timoshenko beams, Composite Structures 96 (2013) 121-134.

18. I.V. Andrianov, V.V. Danishevs'kyy, B. Markert, Nonlinear vibrations and mode interactions for a continuous rod with microstructure, Journal of Sound and Vibration 351 (2015) 268-281.

19. I.V. Andrianov, V.V. Danishevs'kyy, H. Topol, D. Weichert, Homogenization of a 1D nonlinear dynamical problem for periodic composites, ZAMM - Journal of Applied Mathematics and Mechanics / Zeitschrift für Angewandte Mathematik und Mechanik 91 (2011) 523 - 534.

20. I.V. Andrianov, V.V. Danishevs'kyy, O.I. Ryzhkov, D. Weichert, Dynamic homogenization and wave propagation in a nonlinear 1D composite material, Wave Motion 50 (2013) 271-281.

21. M.I. Hussein, G.M. Hulbert, R.A. Scott, Dispersive elastodynamics of $1 \mathrm{~d}$ banded materials and structures: analysis, Journal of Sound and Vibration 289 (2006) 779-806.

22. A. Som, I. Chakraborty, T.A. Maark, S. Bhat, Th. Pradeep, Cluster-mediated crossed bilayer precision assemblies of 1D nanowires, Advanced Materials 28(14) (2016) 2827-2833.

23. A.I. Lur'e, Nonlinear Theory of Elasticity, North-Holland, Amsterdam, 1990.

24. F.D. Murnaghan, Finite Deformation of an Elastic Solid, John Wiley and Sons, Inc, New York, 1951.

25. L. Landau, G. Rumer, Über Schallabsorption in festen Korpern, Physikalische Zeitschrift der Sowjetunion 11 (1937) 18-23.

26. W. Voigt, Über eine anscheinend notwendige Erweiterung der Theorie der Elasticität, Nachrichten von der Koniglichen Gesellschaft der Wissenschaften und der Georg-AugustsUniversitat zu Gottingen (1893) 534-552.

27. J. Fish, W. Chen, Space-time multiscale model for wave propagation in heterogeneous media, Computer Methods in Applied Mechanics and Engineering 193 (2004) 4837-4856.

28. J.D. Kaplunov, A.V. Pichugin, On rational boundary conditions for higher-order long-wave models, IUTAM Symposium on Scaling in Solid Mechanics. IUTAM Bookseries 10 (2009) 8190.

29. A.B. Movchan, N.V. Movchan, C.G. Poulton, Asymptotic Models of Fields in Dilute and Densely Packed Composites, Imperial College Press, London, 2002.

30. G. Carta, M. Brun, A dispersive homogenization model based on lattice approximation for the prediction of wave motion in laminates, Journal of Applied Mechanics 79 (2012) 021019-1 0211019-8. 
31. M.P. Mortell, R.B. Seymour, Pulse propagation in a nonlinear viscoelastic rod of finite length, SIAM Journal on Applied Mathematics 22(2) (1972) 209-224.

32. E.M. Daya, B. Braikat, N. Damil, M. Potier-Ferry, Continuum modeling for the modulated vibration modes of large repetitive structures, C. R. Mecanique 330 (2002) 333-338.

33. E.M. Daya, M. Potier-Ferry,Vibrations of long repetitive structures by a double scale asymptotic method, Structural Engineering \& Mechanics 12(2) (2001) 215-230.

34. R.V. Craster, J.D. Kaplunov, A.V. Pichugin, High frequency homogenization for periodic media, Proceedings of the Royal Society A: Mathematical, Physical and Engineering Sciences 466 (2010) 2341-2362. 